\title{
A Simple Method for Analysing Binary Data
}

\author{
By S. P. LAPAGE AND W. R. WILLCOX \\ National Collection of Type Cultures, Central Public Health \\ Laboratory, Colindale Avenue, London, $\mathrm{NW}_{9}{ }_{5} \mathrm{HT}$
}

(Received 6 May 1974; revised I2 August 1974)

\section{INTRODUCTION}

A simple procedure was needed to sort and assess accumulated test results on numbers of bacterial strains. The method described helps to point out typical and aberrant strains; strains that give identical results are readily detected; and all the observed reaction patterns are represented in a condensed form.

\section{METHOD}

The data consist of the binary ( + or - ) results of tests applied to strains of bacteria; the strains are divided into groups, e.g. species. Table I gives a set of data for Actinobacillus lignieresi. The method is as follows:

(I) Count for each test the numbers of + and - results. Indicate if the test is consistently + or - throughout; if not, enter the number and type of the less common test results (Table I).

(2) Remove any tests which are consistently + or - throughout (Fig. I $a$ and $b$ ). Then list the remaining tests vertically in order of the number of their less common test results beginning with the lowest, i.e. first tests in which only one strain was $+($ or -$)$, then tests in which two strains were + (or - ), and so on (Fig. I $c$ and $d$ ).

(3) Take the first test in the list (Fig. I c), find the strain or strains "giving the less common result in this test, and write down the strain numbers and the results given by these strains in all the listed tests. In the example (Fig. I $e$ ) strain I I is plotted first as it is the only strain with a negative result in the first test, i.e. nitrate reduction.

(4) Take the next test in the list (Fig. I $c$ ), find the strains giving the less common result in this test, and write down the results of any of these strains not already plotted. This is repeated for the tests in order until all the strains have been plotted, or the last test is reached in which case any remaining strains are then plotted. In the example (Fig. I $e$ ), the strain giving a negative result in the second test, maltose, has already been plotted, strain I ; and strain 12 is the next to be plotted as it is the only strain giving a positive result in the third test, raffinose.

Thus each test 'selects' from the strains that have not already been plotted, those which give the less common result in that test. Figure $\mathrm{I}(e)$ shows that the results for the strains selected by any test need not be plotted for previous tests as the strains conform to the typical pattern in these tests. A line can be drawn to show the typical test results, as in Fig. I (e). If more than one strain is selected by a test, the results of the strains selected are compared in the remaining tests to see whether any of the strains give identical results. This can be done by making a temporary plot of each strain, comparing it with the strains already plotted, and entering the strain only if it has a new pattern of results. Strains found to have identical patterns are noted, as in Fig. I $(f)$. Finally, it may be helpful to 
Table I. Example set of data: test results for $\mathrm{I} 3$ strains of Actinobacillus lignieresi

Less

Strain numbers

Consistent common

Motility at $37^{\circ} \mathrm{C}$

$\begin{array}{lllllllllllllll}\text { I } & 2 & 3 & 4 & 5 & 6 & 7 & 8 & 9 & \text { IO } & \text { II } & \text { I } 2 & \text { I3 } & \begin{array}{c}\text { results } \\ \text { rest }\end{array} & \begin{array}{c}\text { test } \\ \text { results }\end{array}\end{array}$

test test

Motility at RT

Growth at $37^{\circ} \mathrm{C}$

Growth at RT

Pigment production

MacConkey agar, growth

Catalase, presence

Oxidase, presence

Hugh \& Leifson test, fermentative

Nitrate reduction

Indole production

Methyl red at $37^{\circ} \mathrm{C}$

Methyl red at RT

Voges-Proskauer at $37^{\circ} \mathrm{C}$

Voges-Proskauer at RT

Simmons' citrate, growth

Christensen's citrate, alkali

production

Urease, presence

KCN medium, growth

$\mathrm{H}_{2} \mathrm{~S}$ production (lead acetate

paper)

$\mathrm{H}_{2} \mathrm{~S}$ production (TSI)

Gluconate reduction

Malonate utilization

$\beta$-Galactosidase production

Phenylalanine deamination

Arginine, dihydrolase

Lysine, decarboxylase

Ornithine, decarboxylase

Glucose, acid production

Glucose, gas production

Production of acid from:

Adonitol
Arabinose
Cellobiose
Dulcitol
Glycerol
Inositol
Lactose
Maltose
Mannitol
Raffinose
Rhamnose
Salicin
Sorbitol
Sucrose
Trehalose
Xylose
Starch

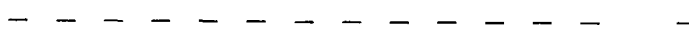

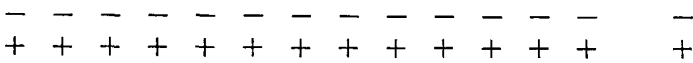

$++-++-------$

$--\infty-----$

$+++++++++++++$

$++-++-++++++$

$----+----\infty+$

$+$

$\frac{-}{+}$

$4+$

$----++++++++$

$++++++++++-++$

$---------$

$-----------$

$-----\ldots--$

$---------$

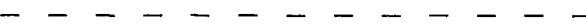

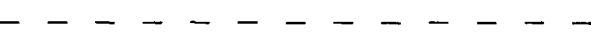

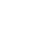

$2+$

$5-$

I-

$---+++-+++++$

$5-$

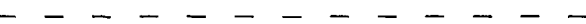

$-$

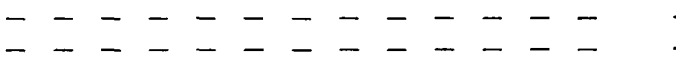

$----------$

$+++++++++++++t$

$---------$

$----\quad-\quad-\quad----$

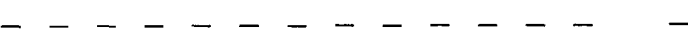

$--\overline{-}-\overline{-}-\overline{-}-\overline{-}-\overline{+}$

$++++++++++++t+$

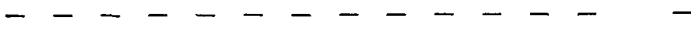

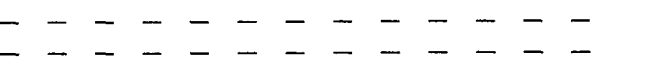

$-----------$

$-----------$

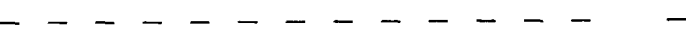

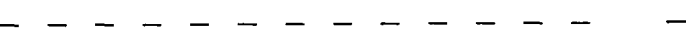

$----------$

$++++++++++-++\quad \mathrm{r}-$

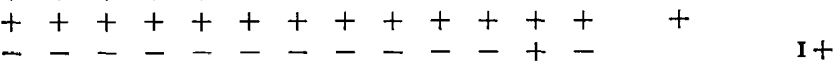

- - - - - - - - - - - - $-\overline{-}$

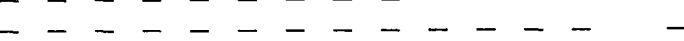

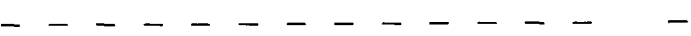

$++++++++++++++$

$-----------1+$

$++++++++++++++$

$-----\cdots--$

Selenite reduction
Gelatin liquefaction within 5 days -------+-

$2+$

Gelatin liquefaction within 5 days ------
Gelatin liquefaction within 28 days -----

$\mathrm{RT}$, room temperature (approximately 14 to $27^{\circ} \mathrm{C}$ ) or incubator at 22 or $30^{\circ} \mathrm{C}$. TSI, triple sugar iron agar. 
(a) All strains are positive in: Growth at $37^{\circ} \mathrm{C}$ Glucose, acid production Xylose, acid production

(b) All strains are negative in: Motility at $37^{\circ} \mathrm{C}$

Indole production

Voges-Proskauer at $37^{\circ} \mathrm{C}$

Christensen's citrate, alkali production

$\mathrm{H}_{2} \mathrm{~S}$ production (TSI)

Phenylalanine deamination

Ornithine, decarboxylase

Arabinose, acid production

Glycerol, acid production

Rhamnose, acid production

Trehalose, acid production

(c)

Nitrate reduction

Maltose, acid production

Raffinose, acid production

Catalase, presence

Oxidase, presence

Selenite reduction

Growth at RT

Hugh \& Leifson test, fermentative

Urease, presence
MacConkey agar, growth

Mannitol, acid production

Gelatin liquefaction within 28 days Pigment production

Motility at RT

Methyl red at $37^{\circ} \mathrm{C}$

Voges-Proskauer at RT

KCN medium, growth

Gluconate reduction

Arginine, dihydrolase

Glucose, gas production

Cellobiose, acid production

Inositol, acid production

Salicin, acid production

Starch, acid production
$\beta$-Galactosidase production

Sucrose, acid production
Methyl red at RT

Simmons' citrate, growth

$\mathrm{H}_{2} \mathrm{~S}$ production (lead acetate paper)

Malonate utilization

Lysine, decarboxylase

Adonitol, acid production

Dulcitol, acid production

Lactose, acid production

Sorbitol, acid production

Gelatin liquefaction within 5 days

(e) Strain numbers

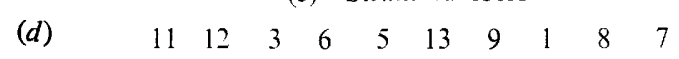

(f) Identical strains are: I, 2 and $4 ; 7$ and 10.

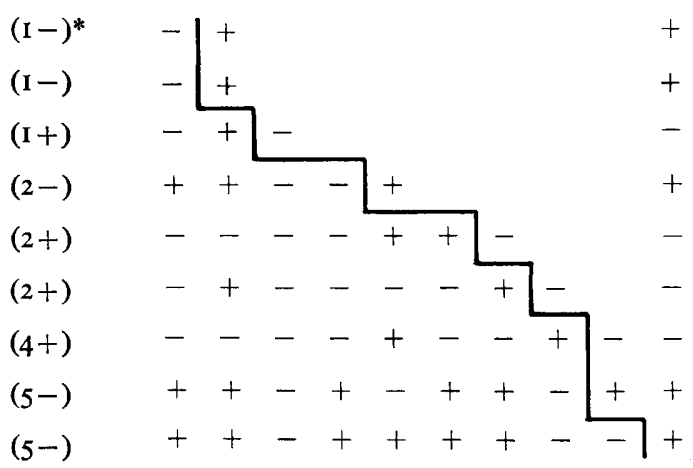

Fig. I. The example set of data (Table I) plotted by the method described. RT, room temperature (approx. 14 to $27^{\circ} \mathrm{C}$ ) or incubator at 22 or $30^{\circ} \mathrm{C}$. TSI, triple sugar iron agar. ${ }^{*}$ The entry in brackets is the number and type of the less common results for each test. $(a)$ to $(f)$ refer to the stages of the method described in the text.

re-arrange some of the earlier tests and strains (Fig. 2) so that any strains which give unusual results in several tests are made more prominent. In Fig. $2(a)$ strain 24 is seen to be aberrant in three tests so it is moved to the first position and the tests in which it is aberrant are brought together as the first three tests (Fig. $2 b$ ).

\section{RESULTS AND DISCUSSION}

The method indicates the tests in which all members of the taxon give consistent results; these are obviously possible tests to use in characterizing the taxon and in identification keys. The plot itself represents all the test result patterns in a condensed form and strains which give identical results are economically detected. The plot orders the strains, to some extent, according to how typical they are of the taxon as a whole; the most aberrant strains are likely to be found to the left of the plot, e.g. strain I I in Fig. I (e), and the most typical to the right. 
(a)

Motility at $37^{\circ} \mathrm{C}$

Pigment production

Methyl red at $37^{\circ} \mathrm{C}$

$\beta$-Galactosidase production

Arginine, dihydrolase

Lysine, decarboxylase

Glucose, gas production

Etc.

(b)

Pigment production

Arginine, dihydrolase

Glucose, gas production

Motility at $37^{\circ} \mathrm{C}$

Methyl red at $37^{\circ} \mathrm{C}$

$\beta$-Galactosidase production

Lysine, decarboxylase

Etc.
Strain numbers

$\begin{array}{llllll}2 & 24 & 10 & 9 & 3 & \text { etc. }\end{array}$

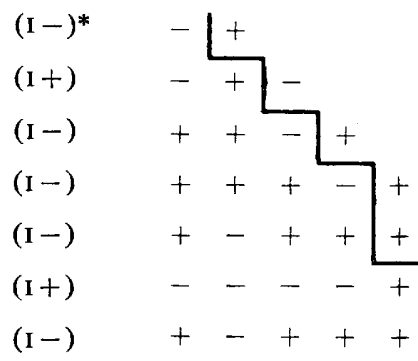

$\begin{array}{llllll}24 & 2 & 10 & 9 & 3 & \text { etc. }\end{array}$

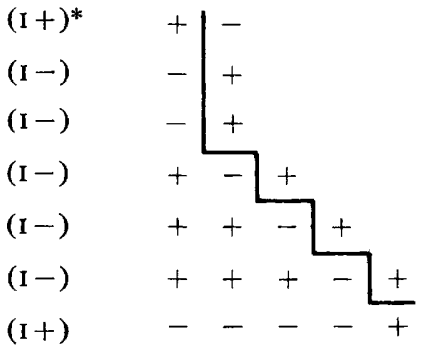

Fig. 2. The first few tests and strains of a plot for Citrobacter koseri showing re-arrangement to make an aberrant strain (No. 24) more prominent: (a) original arrangement determined by the order in which the tests were considered; $(b)$ re-arrangement. *The entry in brackets is the number and type of the less common test results for each test.

Several methods have been proposed for defining typical organisms for taxa (Sneath \& Sokal, I973, p. 195). The 'hypothetical median organism' (Liston, Weibe \& Colwell, 1963) is the organism giving the most common result in each test, termed 'hypothetical' because it can be used in numerical procedures even if no corresponding actual organism is found. The centrotype (Silvestri, Turri, Hill \& Gilardi, 1962) is the organism with the highest mean similarity to the other organisms in the taxon. If a median organism does exist it will also be the centrotype provided that the simple matching coefficient (Sneath \& Sokal, I973, p. I32) is used as a measure of similarity. If there is a median organism in a taxon which is examined by the present method it will always appear as the last (rightmost) strain of the plot, but if there is no median organism the last strain will not necessarily be the centrotype. Plots were made for 56 taxa of Gram-negative fermentative bacteria (Bascomb, Lapage, Curtis \& Willcox, I973); the number of strains per taxon varied from 2 to 149 and all I 200 strains were tested in the 50 tests listed in Table I. Taxa with only two strains were ignored and of the remaining 53 taxa a median organism was found in $36(68 \%)$, the last strain plotted was the centrotype in a further $15(28 \%)$ and the centrotype was one of the last two or three strains, easily recognizable by inspection, in the remaining two $(4 \%)$. There was no significant correlation between the occurrence of median organisms and the number of strains in the taxa.

The method is particularly suited to manual working because it reduces the number of test results which must be transcribed, but for convenience in this laboratory a computer 
program has been prepared and shown to operate satisfactorily. As a computer procedure the method would seem to be an efficient way of detecting identical patterns of test results and locating centrotypes.

We wish to thank the Department of Health and Social Security for the grant which enabled us to develop the computer program.

\section{REFERENCES}

BASCOMB, S., LAPAGE, S. P., CURTIS, M. A. \& WiLLCoX, W. R.(I973).Identification of bacteria by computer: identification of reference strains. Journal of General Microbiology 77, 29I-3I5.

Liston, J., WeiBe, W. \& Colwell, R. R. (1963). Quantitative approach to the study of bacterial species. Journal of Bacteriology 85, 1061-1070.

Silvestri, L. G., TurRi, M., Hill, L. R. \& Gilardi, E. (1962). A quantitative approach to the systematics of actinomycetes based on overall similarity. In Microbial Classification: Twelfth Symposium of the Society for General Microbiology, pp. 333-360. Edited by G. C. Ainsworth and P. H. A. Sneath. Cambridge University Press.

SNeAtH, P. H. A. \& Sokal, R. R. (1973). Numerical Taxonomy. San Francisco: W. H. Freeman. 